\title{
Corporeizar las etnografías desde perspectivas feministas
}

\author{
Lola Martínez Pozo' (iD 0000-0001-7897-2676 \\ 'Universidad de Granada. Granada. España. 18010. tsocial@ugr.es
}

\section{$-$}

Resumen: En este artículo propongo una reflexión sobre el lugar de los cuerpos en la producción de conocimientos. En la tarea de repensar cuestiones epistemológicas, metodológicas y políticas orientadas a corporeizar las etnografías y desarrollar una antropología encarnada, en estas páginas destaco diversas perspectivas socio-antropológicas y feministas críticas que me han permitido experimentar la práctica etnográfica como corporal. A continuación, comparto algunas reflexiones procedentes de la experiencia etnográfica sobre la politización de los cuerpos y las tecnologías desde perspectivas (trans)feministas y prácticas hacking, donde conecto los feminismos, la etnografía feminista y los proyectos (trans)feministas compartidos por Ixs entrevistadxs, en tanto genealogías diferenciadas dirigidas a hackear los códigos normativos de producción de conocimiento y a desarrollar conocimientos de resistencia desde las experiencias corporales.

Palabras Clave: cuerpos, feminismos, conocimiento antropológico, etnografía feminista.

\section{Embodying Ethnographies from Feminist Perspectives}

Abstract: In this article, I propose a discussion regarding the place of bodies in the production of knowledge. In the task of rethinking epistemological, methodological and political issues oriented towards embodying ethnographies and developing an embodied anthropology, in these pages I highlight some socio-anthropological and critical feminist perspectives that have allowed me to consider and experience the ethnographic practice as a corporal practice. I also share some considerations of the ethnographic experience on the politicization of bodies and technologies from (trans)feminist perspectives and hacking practices, where I connect feminisms, feminist ethnography and the (trans)feminist projects shared by the activists as differentiated genealogies aimed at hacking normative codes and developing resistant knowledge from bodily experiences.

Key Words: Bodies; Feminisms; Anthropological Knowledge; Feminist Etnography.

\section{Corporalizar as etnografias a partir das perspectivas feministas}

Resumo: No presente artigo, proponho uma reflexão sobre o lugar dos corpos na produção dos conhecimentos. Na tarefa de repensar questões epistemológicas, metodológicas e políticas orientadas a corporalizar as etnografias e a desenvolver uma antropologia corporalizada, destaco diversas perspectivas sócio antropológicas, bem como perspectivas feministas críticas, que me permitiram experimentar a prática etnográfica como corporal. A seguir, compartilho algumas reflexões advindas da experiência etnográfica sobre a politização dos corpos e das tecnologias a partir de perspectivas (trans)feministas e de práticas de hacking, onde relaciono feminismos, etnografia feminista e projetos (trans)feministas compartilhados pelos entrevistados como genealogias diferenciadas que visam hackear os códigos normativos da produção de conhecimento e desenvolver conhecimento de resistência a partir de experiências corporais.

Palavras-chave: corpos; feminismos; conhecimento antropológico; etnografia feminista.

\section{Introducción}

En este trabajo parto de una reflexión sobre el lugar de los cuerpos en la construcción del conocimiento antropológico desde la conexión entre las genealogías teóricas sobre el cuerpo en antropología social y los feminismos con la propia experiencia etnográfica. Se trata de abordar 
enfoques críticos dirigidos a repensar procesos de investigación desde perspectivas y prácticas políticas que permitan conocer, aprehender y narrar desde los cuerpos.

Siguiendo la propuesta de "practicar una antropología de y desde los cuerpos en la que las palabras ya no tengan que ocultar la carne que les dio vida" (Silvia CITRO, 2010, p.18), en estas líneas planteo el desplazamiento desde los cuerpos como ámbito de estudio hacia las experiencias corporales como procesos de construcción de conocimiento en el marco de los feminismos, la antropología y la etnografía feminista.

En este sentido, perspectivas críticas feministas en investigación social (Teresa DE LAURETIS, 1986; Sandra HARDING, 1987, 1988, 1996 [1986]; Donna HARAWAY, 1988, 1995 [1991]; Carme ADÁN, 2006; Jacqueline WATTS, 2006; María Teresa MARTíN; José María MUÑOZ, 2014; Norma BLÁZQUEZ, 2017) han cuestionado los modelos hegemónicos de construcción y validación del conocimiento científico insertos en mecanismos de poder androcéntricos y patriarcales. A estas perspectivas se suman enfoques críticos de la subalternidad, en relación a la producción de conocimiento en ámbitos académicos y disciplinarios inscritos en esquemas de dominación política, económica y cultural neo-coloniales, que subrayan la urgencia de cuestionar las perspectivas universalizantes y hegemónicas en el interior de los propios feminismos mediante la articulación de las críticas feministas y decoloniales (Gayatri C. SPIVAK, 2003 [1985]; bell HOOKS ef al., 2004; Ochy CURIEL, 2007, 2018 [2014]; Chandra TAPALDE MOHANTY, 2008 [1984]; Liliana SUÁREZ NAVAZ; Rosalía HERNÁNDEZ CASTILLO, 2008; María LUGONES, 2010; Yuderkys ESPINOSA, 2014; ESPINOSA; Diana GÓMEZ; Karina OCHOA, 2014).

Desde las anteriores perspectivas críticas feministas, en su heterogeneidad y divergencias, considero que se han desvelado, enfatizado e incorporado los cuerpos, las emociones, las experiencias y subjetividades en la investigación social. No obstante, desde mi punto de vista, prosiguen los desafíos en cuanto al lugar de las experiencias corporales en la producción de conocimiento antropológico y etnográfico, los cuales me sugieren la importancia de repensar cuestiones epistemológicas, metodológicas y políticas orientadas a corporeizar nuestras etnografías y a desarrollar una antropología más encarnada.

En mi proceso de investigación, repensar y experimentar los cuerpos como lugares de construcción de conocimientos me ha implicado rescatar aquellos enfoques críticos que han reivindicado lo corporal como locus epistemológico, metodológico y como ámbito político.

Por ello, en este artículo y en primer lugar, destaco una serie de aproximaciones socioantropológicas y feministas al cuerpo que, desde mi perspectiva, han contribuido a poner de relieve la centralidad de las corporalidades en los ámbitos académicos y políticos. A continuación, comparto algunas reflexiones procedentes de la experiencia etnográfica con trayectorias y proyectos de disidencia corporal y tecnológica desde perspectivas (trans)feministas y prácticas hacking, que me han potenciado a relacionar críticamente cuerpo, tecnología y conocimiento.

Por último, finalizo, con una relectura de los (trans)feminismos -en tanto perspectivas y prácticas críticas de ruptura y reprogramación de los códigos normativos que conforman nuestros cuerpos, sexualidades, subjetividades y tecnologías - en conexión con las genealogías críticas que nos aportan las perspectivas feministas en investigación social y la etnografía feminista, en la medida en que interpreto dicha vinculación como un engranaje enriquecedor desde donde repensar cuestiones epistemológicas y metodológicas en torno al lugar de nuestros cuerpos y experiencias corporales en la producción de conocimientos.

\section{Genealogías teóricas corporales}

Considero que reformular los cuerpos en la producción de conocimiento antropológico implica desplazamientos y retos en los que identifico se hallan inmersos los feminismos y diferentes aproximaciones socio-antropológicas.

En este sentido, desde mi experiencia investigadora contemplo que los feminismos, en tanto corpus teóricos y políticos diversos, se constituyen como una amplia genealogía que a continuación interpreto como un conjunto de epistemologías, metodologías y políticas somáticas donde las corporalidades emergen en la producción de conocimientos.

Por su parte, en las ciencias sociales y en la disciplina antropológica, a partir de la década de los ochenta, comienzan a adquirir relevancia ciertas perspectivas críticas y fenomenológicas donde los cuerpos transcienden la categoría de objeto de investigación o ámbito de estudio y son configurados como sujetos de investigación y conocimiento.

Asimismo, este giro epistemológico y metodológico que permite enfatizar lo corporal viene de la mano de diversas contribuciones epistemológicas y metodológicas feministas.

Por ello, en este trabajo indago en diferentes genealogías socio-antropológicas y feministas que han desarrollado aproximaciones al cuerpo reivindicándolo como locus epistemológico, metodológico y político. Al respecto, la intención no es abarcar de forma exhaustiva la heterogeneidad de propuestas, sino más bien, subrayar aquellas que han tenido una especial relevancia en mi proceso de investigación. 


\section{Feminismos: lo corporal es personal, político y teórico}

Desde los feminismos se han desarrollado diversas epistemologías sobre el cuerpo, que implican, a su vez, diferentes formas de acción, transformación y resistencia (Mari Luz ESTEBAN, 2011 ), por ello, considero que son una genealogía amplia e ineludible de aportaciones a indagar sobre el lugar de las corporalidades en la producción de conocimientos.

Elizabeth Grosz (1994) nos presenta cómo el cuerpo ha sido devaluado dentro de la 'teoría feminista occidental' dominante debido a la adopción acrítica de asunciones patriarcales vinculadas con el pensamiento dualista, la filosofía racionalista y el sujeto universal y neutral de la modernidad.

La jerarquización occidental de la oposición cuerpo/mente y su asociación con el dualismo femenino/masculino que ha vinculado culturalmente a las mujeres con la corporalidad, llevó a las emergentes vindicaciones feministas de la época moderna a replantear y romper la conexión de las mujeres con lo corporal y con el determinismo biológico al que se asociaban sus discriminaciones (Marta AZPEITIA, 2001).

No obstante, la corporalidad siempre ha constituido un lugar central en los análisis, teorizaciones y prácticas políticas feministas, ya que la construcción sociocultural del género involucra materialmente al cuerpo (ESTEBAN, 2004a). Precisamente, una de las grandes contribuciones feministas ha sido colocar el cuerpo en el núcleo de la política. Por ello, el vínculo entre corporalidad y poder ha impulsado desarrollos políticos, teóricos y prácticos al respecto, especialmente desde los feminismos de la década de los setenta (Marta LAMAS, 2002).

El cuerpo, por tanto, se ha constituido en epicentro de producción teórica y políica para las heterogéneas perspectivas feministas, las cuales nos ofrecen múltiples aproximaciones donde podemos bosquejar convergencias y divergencias.

Para los feminismos de finales del siglo XX el cuerpo reproductivo y sexual era núcleo de reivindicación, así como eje central de análisis y teorizaciones (ESTEBAN, 2004a). De este modo, considero que las concepciones y reivindicaciones del cuerpo político feminista como sexual contribuyeron a la disolución de las fronteras naturaleza/cultura y mente/cuerpo, inaugurando, de esta forma, luchas políticas y producciones teóricas desde los cuerpos y experiencias de las mujeres.

Siguiendo a Grosz (1994), Azpeitia (2001) y Esteban (2004a), podemos trazar un distinción entre los cuatro enfoques más influyentes en relación al cuerpo en el marco de los feminismos occidentales contemporáneos: perspectivas naturalistas vinculadas con las teorías feministas de la igualdad, perspectivas constructivistas desarrolladas por las teorías del género, perspectivas de la diferencia sexual elaboradas desde los feminismos de la diferencia y perspectivas posestructuralistas articuladas por feminismos posmodernos.

Desde posiciones feministas de corte igualitario, el cuerpo es planteado como sexual y biológicamente determinado, reproduciendo visiones esencialistas sobre la especificidad corporal de las mujeres como un obstáculo para acceder a la igualdad. En esta línea, Grosz (1994) destaca a autoras como Simone de Beauvoir, Shulamith Firestone, Mary Wollstonecraft y corrientes como el feminismo liberal e, incluso, el ecofeminismo que, aunque desarrolla visiones más positivas de la particularidad de las experiencias y cuerpos de las mujeres, mantiene asunciones de la corporalidad como natural.

Por su parte, el constructivismo social es una perspectiva ampliamente extendida tanto en ciencias sociales como en los ámbitos académicos feministas, donde la corporalidad es entendida como ente pre-cultural que posteriormente vendrá a ser moldeada por la cultura y la sociedad. Dicho enfoque, aunque se opone a las anteriores perspectivas esencialistas, se inserta en las teorías del género, las cuales reconsideran el cuerpo en base a las categorías dicotómicas de sexo/género. En este sentido, podríamos destacar algunos referentes como Juliet Mitchell, Julia Kristeva, Nancy Chodorow, feministas psicoanalíticas y feministas marxistas (GROSZ, 1994).

Otro enfoque es el desarrollado por los feminismos de la diferencia, donde, en acuerdo con Grosz (1994), destacan figuras como Hélène Cixous, Luce Irigaray, Julia Kristeva y Monique Wittig. En esta línea, el cuerpo es considerado como un objeto social, cultural, político e histórico configurado en el orden del deseo, la significación y el poder, así como, crucial para entender la existencia psicológica y social de las mujeres (GROSZ, 1994). Al respecto, Esteban (2004a) identifica las perspectivas del feminismo de la diferencia como ineludibles al inaugurar formas alternativas para hablar desde el cuerpo.

Por otro lado, corrientes feministas posestructuralistas desarrollan enfoques críticos dirigidos a la ruptura de los dualismos y a la desnaturalización del cuerpo, el sexo, la sexualidad y la identidad, superando, de este modo, los enfoques feministas esencialistas y constructivistas. De esta forma, el cuerpo es entendido como una construcción sociocultural y superficie de inscripción discursiva y material de dispositivos de poder.

En este último sentido, desde finales de la década de los ochenta emergen discursos críticos que han proporcionado perspectivas muy fructíferas sobre la producción corporal a partir 
de la noción foucaultiana de biopoder, como los desarrollados por Teresa De Lauretis (1989), Judith Butler (2007 [1990]), Donna Haraway (1985, 1995), Anne Fausto-Sterling (2006 [2000]) o Paul Preciado (2008).

En esta línea, Fausto-Sterling (2006 [2000]) proporciona un análisis de la construcción de las corporalidades en la modernidad a partir de la producción de los cuerpos sexuados y la política de género en las sociedades occidentales.

Asimismo, destaca el trabajo de De Lauretis (1989) que permitió habilitar la noción de tecnologías del género para abordar las corporalidades.

Por su parte, Haraway (1995 [1991]) propone la figuración cyborg como híbrido entre máquina y organismo, entre realidad social y ficción, entre materia de ficción y experiencia, que abarca nuestra realidad social y corporal, cuenta con una potencialidad política de transformación, desconfía del holismo y se sitúa del lado de la parcialidad. Las formulaciones críticas desarrolladas por la autora han inspirado innumerables elaboraciones teóricas y políticas, en ese sentido, la propuesta cyborg en conexión con sus planteamientos sobre el conocimiento situado, también, podría ser muy enriquecedora para repensar el lugar de los cuerpos y las experiencias corporales en la producción de conocimiento antropológico, ya que, parafraseando a la autora, el mito del cyborg consiste en fronteras transgredidas y fusiones a explotar como parte de un necesario trabajo políico (HARAWAY, 1995 [1991]).

Igualmente, la teoría queer ha implicado una ruptura con las genealogías feministas naturalistas, constructivistas y de la diferencia sexual, anteriormente mencionadas, que abordan la corporalidad desde el esencialismo de la diferencia sexual y/o a través de las construcciones identitarias normativas, en este sentido, destacan diversas precursoras de la teoría queer como Butler (2007 [1990], 2002 [1993]) y Eve Kosofsky Sedgwick (1998 [1990], 1994).

Concretando en el trabajo de Butler (2002 [1993]), desde mi punto de vista, éste ha sido una aportación ineludible en torno a la materialidad del cuerpo en los análisis sociales y feministas mediante sus teorizaciones sobre la construcción del cuerpo, el género y la sexualidad a través de la noción de performatividad. La autora aborda la producción sociocultural del cuerpo como superficie de inscripción, en torno a la articulación entre sexo-género y heterosexualidad obligatoria a través de la performatividad como discursos y prácticas de referencia, reproducción y reiteración de la norma. Asimismo, destaca la intersección del género, la sexualidad, la raza y otros ejes de poder en la materialización de los cuerpos, en la construcción de identidades normativas y excluidas, y en la producción de cuerpos viables y aquellos que no lo son.

Sus análisis sobre la materialidad del sexo, del cuerpo, y sus críticas hacia la distinción entre sexo/género predominante en las genealogías feministas occidentales, permitieron problematizar la categoría mujeres como sujeto político feminista anclada en un corporalidad biológica común a todas (BUTLER, 2007 [1990]), así como desarrollar propuestas en torno a las desidentificaciones colectivas para reconceptualizar cuáles son los cuerpos que importan y cuáles son aquellos cuerpos que han de surgir como materia crítica (BUTLER, 2002 [1993]).

En este último sentido propuesto por Butler, a estas cuatro aproximaciones feministas al cuerpo podríamos añadir un quinto enfoque que se corresponde con las perspectivas feministas, donde el cuerpo se contempla en términos culturales e históricos como agente, es decir, como lugar de contestación y resistencia (ESTEBAN, 2004a).

Tras este recorrido por algunas de las perspectivas feministas sobre la corporalidad, considero que, no exclusivamente, sitúan lo corporal en la producción de conocimiento vinculando vida, cuerpo y pensamiento, sino que también lo articulan como núcleo de reivindicaciones teóricas y políticas, es decir, "pensar desde el cuerpo, 'habitarlo' y con-vivir con él se convierte en un proyecto de revalorización de la alteridad que la teoría feminista nos invita a explorar una y otra vez" (Olaya FERNÁNDEZ GUERRERO, 2012, p. 368).

Por ello, desde mi punto de vista, los feminismos y las teorizaciones feministas pueden ser reconsideradas en función de sus aproximaciones y perspectivas críticas sobre el cuerpo, interpretándolas como un conjunto de discursos, experiencias, epistemologías, metodologías y políticas somáticas donde el cuerpo prolifera en la producción de un conocimiento transformador.

No obstante, considero que prosiguen los retos en la tarea de repensar el cuerpo en el marco de los feminismos. En acuerdo con Esteban (2004a), se precisan enfoques que sean críticos con la normatividad, con las estructuras sociales hegemónicas, pero que también visibilicen las resistencias, incluyan el cuerpo y sus experiencias, así como den cuenta de las lecturas y las prácticas culturales alternativas, desarrollen nuevos modos de hablar desde el cuerpo y nuevas formas políticas y estrategias respecto a lo corporal.

\section{Perspectivas socio-antropológicas de y desde el cuerpo}

Desarrollar una antropología desde el cuerpo, una antropología encarnada, que articule la vida y las experiencias corporales con los procesos de investigación, que parta y hable desde los cuerpos, es una visión minoritaria y periférica, así como una práctica controvertida, ya que 
implica replantear los principios del positivismo predominante. Pero, es una tarea muy necesaria para la disciplina que permite reconocer lo personal y lo subjetivo sin despojarse del propio cuerpo e incluir otras formas de aproximarnos a las realidades sociales en las prácticas académicas (ESTEBAN, 2004b).

Las controversias en torno a la subjetividad como obstáculo no resultan aspectos novedosos en la disciplina. La idea de alcanzar resultados objetivos mediante la elusión de los sesgos personales, los cuerpos y las experiencias ha sido una constante en investigación social que empezó a cuestionarse a partir de la década de los setenta.

En un contexto de crisis de las ciencias sociales y, por ende, del paradigma científico positivista, adquieren relevancia enfoques interpretativistas y múltiples perspectivas que desarrollan una valoración positiva de la subjetividad, donde las experiencias, vidas e investigación se entrelazan y son consideradas fuente de conocimiento (Mariana DEL MÁRMOL et al., 2008).

En este sentido, desde la antropología posmoderna se ha enfatizado el carácter múltiple, plural y fragmentario de los discursos insistiendo en la relevancia de lo personal y la reflexividad en el trabajo de campo y la escritura etnográfica. Asimismo, diversos enfoques teóricosmetodológicos como la vinculación de corrientes posestructuralistas y fenomenológicas, la antropología del cuerpo y la autoetnografía han desarrollado las posibilidades del conocimiento cuando este parte del propio cuerpo y de las propias experiencias (DEL MÁRMOL et al., 2008).

En dicho contexto de deconstrucción de las grandes narrativas se empiezan a legitimar una multiplicad de perspectivas procedentes de una pluralidad de voces que suponen la incursión de conocimientos situados, locales, periféricos, fronterizos y marginados en los ámbitos académicos (Joel FELIU, 2007). Desde mi perspectiva, dicha ocupación de ámbitos ilegítimos hasta el momento también hizo estallar los efectos del género, la sexualidad, la raza, las migraciones, la clase, la diversidad funcional, etc., en la producción de conocimiento, es decir, evidenció la materialidad de los cuerpos, las trayectorias, experiencias corporales y las relaciones de poder en los procesos de construcción de conocimiento.

De acuerdo con Citro (2010), repensar el lugar de los cuerpos en la construcción del conocimiento antropológico desde nuestras prácticas etnográficas implica reflexionar sobre la complejidad de las realidades sociales desde aquello que les acontece a las corporalidades de las personas y, para ello, es necesario conocer y dar cuenta de las genealogías que desde la década de los setenta plantean debates y enfoques críticos al respecto.

En este sentido, la teoría social del cuerpo es un enfoque teórico-metodológico reciente, aunque deudora de trabajos predecesores, donde la corporalidad se torna en "nudo de estructura y acción y, en centro de reflexión social y antropológica" (ESTEBAN, 2004a, p. 19). Por su parte, la antropología del cuerpo comienza a definirse y configurarse a partir de la década de los setenta en torno a enfoques que plantean las dimensiones sociales, culturales e históricas de nuestras corporalidades, donde éstas pasan a concebirse como construcciones socioculturales (ESTEBAN, 2004a).

En el marco de las diferentes perspectivas socio-antropológicas del cuerpo, encontramos múltiples formas de constituirlo como objeto de estudio. No obstante, a pesar de dicha heterogeneidad, tomando en consideración a Del Mármol y Sáez (201 1), en la formulación de la corporalidad como ámbito de estudio en ciencias sociales podemos distinguir tres enfoques principales: político, simbólico y la perspectiva de embodiment (corporeización). En los enfoques sobre el cuerpo que destacan su carácter político, éste es configurado como espacio de inscripción atravesado por dispositivos de normalización, regulación y control. Por su parte, el enfoque simbólico aborda la corporalidad como ámbito de representación y medio de expresión fruto de sistemas simbólicos culturales. Y, por último, la perspectiva de embodiment supera las tendencias de inscripción políica y simbólica anteriores al destacar el papel central del cuerpo, su carácter activo y transformador de las realidades donde, basándose en la noción ser-en-el-mundo formulada por Merleau-Ponty, propone una aproximación fenomenológica del cuerpo vivido que supera la noción de objeto de estudio y lo presenta como punto de partida metodológico.

Desde la antropología se han desarrollado múltiples reflexiones en torno al cuerpo. Debido al carácter de este artículo, en estas líneas destaco algunos de mis referentes que, interpreto, han contribuido a la configuración de una antropología más encarnada.

De este modo, las aproximaciones antropológicas en torno al cuerpo fueron inauguradas por Marcel Mauss (1979 [1971]) y su concepción de técnicas corporales desde las cuales describía cómo la cultura y la sociedad dan forma al cuerpo. Por lo que cuenta con el reconocimiento académico de situarlo como un espacio de análisis y de instigar a las ciencias sociales y a la antropología a su inmersión en un horizonte escasamente abordado.

A pesar de que en los años treinta, con Marcel Mauss, se inauguraran nuevos panoramas para la disciplina en cuanto a las corporalidades, no sería hasta la década de los setenta cuando empieza a configurarse la antropología del cuerpo. En este sentido, el trabajo de Mary Douglas (1988 [1970]) y las compilaciones elaboradas por Jonathan Benthall y Ted Polhemus 
(1975) y por John Blacking (1977) constituyeron el impulso para el desarrollo de reflexiones y miradas contrahegemónicas en torno al cuerpo desde la disciplina antropológica.

Concretando en el trabajo de Douglas (1988 [1970]), la autora desarrolló importantes contribuciones en cuanto a las dimensiones simbólicas de los cuerpos mediante la consideración de los mismos como signos sociales y metáforas de sistemas políticos y socioculturales.

Por otro lado, el trabajo de Foucault (1987 [1976]) sobre la articulación entre biopoder y corporalidad ha tenido una notable influencia en el conjunto de las teorizaciones sociales del cuerpo y en numerosos trabajos antropológicos, superando los enfoques representativistas y simbólicos abordados. Como, por ejemplo, los desarrollados por Nancy Scheper-Hughes y Margaret Lock (1987) en cuanto a la deconstrucción crítica de las asunciones occidentales sobre el cuerpo y sus análisis sobre las diferentes perspectivas a través de las cuales éste puede ser considerado: el cuerpo individual como cuerpo experimentado; el cuerpo social como símbolo para repensar la relación entre naturaleza, cultura y sociedad; y el cuerpo político como artefacto de control social y político. Asimismo, el trabajo de Pierre Bourdieu (1986) sobre la relación entre habitus, cuerpo y clase social también implicó un giro en las aproximaciones socio-antropológicas a la corporalidad como símbolo, inscripción, representación y medio de expresión, enfatizando el cuerpo como locus de prácticas sociales e incluyendo la corporalidad para los agentes sociales. Igualmente, el antropólogo Michael Jackson (2010 [1983]) subrayaba críticamente esta tendencia dominante de los modelos simbólicos y semióticos en los estudios antropológicos del cuerpo hasta la década de los ochenta, los cuales consideran la praxis corporal secundaria a la verbal, e implican una escisión entre el sujeto conocedor y hablante y el cuerpo sin conocimiento. Por ello, desde la articulación de las nociones de cuerpo vivido de Merleau-Ponty y de habitus de Bourdieu, Jackson desarrolla un modelo de análisis que enfatiza en los sujetos como corporeizados y en los entramados de las prácticas corporales en el campo social y el mundo material.

En esta misma línea de ruptura con los enfoques antropológicos dominantes en relación con los cuerpos, destacan las aportaciones de la perspectiva embodiment y modos de atención somática desarrolladas por Thomas J. Csordas (1993). El autor señala una apertura de las teorías antropológicas hacia la fenomenología para superar los paradigmas monolíticos de textualidad y representación, a través de la cual algunxs académicxs, influenciadxs por Merleau-Ponty y sus nociones de carne y ser-en-el-mundo, han desarrollado aproximaciones al cuerpo como vivido, considerándolo un punto de partida metodológico más que un objeto de estudio.

De esta forma, embodiment se constituye como una perspectiva y campo metodológico que aborda la corporalidad como sujeto, como sustrato existencial de la cultura, y las experiencias corporales como punto de partida para analizar las realidades. La perspectiva fenomenológica cultural de embodiment me aproxima a la propuesta que comparto en estas páginas acerca de corporeizar las etnografías y de repensar y hacer antropología desde los cuerpos. Asimismo, la noción de modos somáticos de atención enfatiza el interés en prestar atención a y con el propio cuerpo, así como a los otros cuerpos. De esta manera, los modos somáticos de atención planteados por el autor serían procesos de percepción que permiten situarnos en las experiencias de nuestros cuerpos y de los cuerpos de otros, lo cual puede decirnos algo sobre el mundo y sobre aquellxs que nos rodean (CSORDAS, 1993).

Desde las anteriores rupturas críticas y a partir de la década de los ochenta emergen perspectivas críticas que desarrollan profundas transformaciones en las tendencias dominantes de la antropología en su aproximación a las corporalidades. En dichos enfoques, los cuerpos superan la categoría de objeto en los estudios socioculturales y se constituyen como sujetos de investigación. Por ello, considero que desarrollar investigaciones desde los cuerpos requiere situar nuestros análisis en las corporalidades y repensar y experimentar nuevas aproximaciones metodológicas que puedan dar cuenta de las dimensiones corporales de las experiencias y de los procesos de in-corporar-se y hacer-se-cuerpo (ESTEBAN; Juan Antonio FLORES; Julián LÓPEZ, 2011 ). En este último sentido, la etnografía de y desde los cuerpos (CITRO, 2010), la autobiografía (Judith OKELY, 1992), los itinerarios corporales (ESTEBAN, 2004a), la autoantropología (ESTEBAN, 2004b) y la autoetnografía (FELIU, 2007; Mercedes BLANCO, 2012; Noelia GARCÍA, 2013) son algunas de las propuestas y estrategias metodológicas dirigidas a corporeizar las investigaciones y los conocimientos, así como a poner de relieve la centralidad que ocupan los cuerpos en su relación con otros cuerpos -intercorporalidad-y las experiencias corporeizadas en los procesos de producción de conocimientos (Patricia ASCHIERI; Rodolfo PUGLISI, 2010). Asimismo, desde mi punto de vista, la etnografía feminista es una metodología desarrollada como consecuencia de la politización feminista de la antropología y de las críticas epistemológicas y metodológicas feministas a los mecanismos hegemónicos de investigación social y de construcción de conocimiento científico.

Por ello, Ixs académicxs y antropólogxs feministas contamos con un amplio legado en el desarrollo de ejercicios críticos hacía la producción de conocimiento característica del positivismo. En este sentido, como indicaba al inicio del artículo, la pluralidad de enfoques críticos feministas 
ha rechazado las distinciones entre sujeto-objeto, pensamiento-emociones y personal-político. De igual modo, ha desarrollado fructíferas contribuciones dirigidas a la valorización de los sentimientos, las emociones y las experiencias a través de aproximaciones integradoras y transdisciplinarias al conocimiento que emergen de las vidas concretas.

A nivel metodológico, las epistemologías feministas han abordado las relaciones de explotación y jerarquización presentes en investigación social, y han elaborado metodologías orientadas a desarrollar procesos de investigación igualitarios (Judith STACEY, 1991 [1988]).

No obstante, en cuanto a la etnografía como método de investigación feminista, Stacey (1991 [1988]) destacaba una serie de contradicciones entre los principios feministas y el método etnográfico. Las controversias señaladas por la autora subrayan los peligros de explotación, manipulación, apropiación, representación y desigualdad presentes en la etnografía como proceso y producto.

Tales dilemas han sido ampliamente abordados desde la antropología y etnografía feminista mediante la crítica a la objetividad y neutralidad del conocimiento, el cuestionamiento de la noción y representación de la otredad, la visibilización de las relaciones de poder, y la disolución de las dicotomías objeto/sujeto de investigación y conocimiento, entre otras. De las misma forma, desde enfoques feministas se han desarrollado contribuciones epistemológicas y metodológicas dirigidas a: visibilizar discursos y experiencias subalternas; la inclusión de la posición situada, de la subjetividad, el diálogo, las relaciones sociales y el compromiso ético-político en el proceso de investigación y en la escritura etnográfica; y, en consecuencia, han apostado por propuestas metodológicas que enfatizan la reflexividad y la producción de conocimiento parcial y situado en vinculación con 'otrxs' en el marco de estructuras y relaciones sociales de poder (Carmen GREGORIO GIL, 2006).

En este sentido, considero que la etnografía feminista cuenta con la potencialidad de generar espacios y procesos de producción de conocimientos desde otro lugar, desde la intersubjetividad, los intercambios, los diálogos, la implicación política y desde el reconocimiento y valorización de los cuerpos y las experiencias corporales.

\section{Los cuerpos en la producción de conocimientos feministas de resistencia}

Las reflexiones anteriormente planteadas sobre las genealogías teóricas corporales se hallan motivadas desde mi experiencia etnográfica y desde mi comprensión de la corporalidad como un espacio teórico, político y metodológico que excede la categoría de objeto de estudio, tornándose en un enclave de producción de conocimiento.

A partir de dicha comprensión, inicié mi investigación atendiendo a las conexiones entre cuerpos y tecnologías desde perspectivas (trans)feministas.

La vinculación entre investigaciones feministas, feminismos y tecnologías no es una tarea novedosa. Ya han pasado casi cinco décadas desde los iniciales trabajos en ciencia, tecnología y género. Enfoques derivados desde el empirismo feminista y las perspectivas neomaterialistas, hasta las posiciones posmodernas y las ciberfeministas actuales que subrayan una apropiación crítica de las tecnologías, así como la participación de mujeres y otras diversidades en la generación de nuevos discursos, prácticas y significados críticos y transformadores con las desigualdades presentes en la tecnocultura contemporánea (Inmaculada PERDOMO REYES, 2016).

Las teorías feministas de las tecnologías constituyen una amplía genealogía de contribuciones críticas que provienen y atraviesan la gran diversidad de feminismos, principalmente el feminismo radical-libertario, el liberal, el radical-cultural, el socialista, el poscolonial, los ciberfeminismos o los feminismos queer (Núria VERGÉS BOSCH, 2013). Tales teorizaciones feministas son las expresiones teóricas de diversas movilizaciones y prácticas políticas que desarrollan críticas hacía la tecnociencia y las tecnologías como sistemas de colonización y dominación sexista, heteronormativo, racista, clasista, capitalista y capacitista, y generan prácticas dirigidas a una descolonización y autogestión tecnológica.

Dichos ejercicios adquieren un peculiar impulso y relevancia a partir de la década de los noventa mediante la influencia de los trabajos de Haraway (1995 [1991], 1997) sobre la imaginería cyborg y a través del desarrollo de los ciberfeminismos, tecnofeminismos, los activismos ciberqueer y tecnoqueer.

Más recientemente, desde perspectivas y prácticas políticas (trans)feministas, también se han desarrollado reconceptualizaciones de las nociones tradicionales sobre las tecnologías, la inclusión de prácticas tecnoactivistas, ciberactivistas y hacktivistas, y la vinculación de los ámbitos tecno-maquínicos con los cuerpos y las sexualidades.

En este último contexto, la experiencia etnográfica me ha acercado a espacios, proyectos y trayectorias (trans)feministas que se vinculan con las tecnologías desde ámbitos y temáticas heterogéneas. 
A modo de ejercicio reflexivo y autoetnográfico, me es relevante explicitar mi conexión personal y activista con las disidencias políticas desde las que he investigado. En este sentido, mi posicionamiento político feminista y disidente sexual me llevó a iniciar mi investigación desde las disidencias que considero referentes en mi proceso de politización. De este modo, comencé mi proyecto desde las movilizaciones feministas y lgtbqi y proseguí desde los proyectos transfeministas en los que estaba inmersa. A partir de ahí, mis inquietudes políticas en torno a las articulaciones transfeministas entre disidencias corporales y tecnológicas me llevaron a desarrollar trabajo de campo etnográfico con activistas y proyectos que trabajan sobre hacking corporal para abrir, desvelar y subvertir los códigos socioculturales de programación corporal y subjetiva en relación con el género, la sexualidad, la raza, la migración, la clase, la capacidad, etc. Asimismo, esta experiencia etnográfica me permitió compartir prácticas tecnopolíticas (trans)feministas relativas a la autonomía tecnológica y la autodefensa digital feminista. Ello me orientó a ampliar la etnografía al plano digital para aproximarme a proyectos ciberfeministas y hackfeministas que se dirigen tanto a producir nuestras propias infraestructuras tecnológicas para fortalecer nuestras resistencias frente a estructuras tecnológicas hegemónicas, como a generar estrategias y herramientas de autodefensa feminista para contrarrestar y mitigar las ciberviolencias machistas, racistas, Igtbiqfóbicas y diverso-fóbicas.

Dicha trayectoria de investigación la he desarrollado desde la etnografía feminista en tanto herramienta metodológica politizada que he vivenciado como un proceso de intercambio e incorporación desde mi experiencia personal y política. Por tanto, un proceso que me ha permitido conectar mi trayectoria sexual, corporal y política con las disidencias que indago y reflexiono en mi trabajo de investigación.

En esta línea, Mari Luz Esteban (2004b) denomina antropología encarnada y antropología desde una misma a la conexión explícita de investigación antropológica y experiencia corporal personal. De esta manera, habito la investigación como una forma de autoantropología corporeizada, es decir, desde un enfoque atravesado por la propia experiencia con el que me aproximo y doy cuenta de ciertas disidencias políticas compartidas a partir de las prácticas y las experiencias corporales.

Considero que mi experiencia etnográfica se constituye como un proceso personal de investigación y politización que se ubica en el cruce entre cuerpos, sexualidades y tecnologías desde perspectivas (trans)feministas y prácticas hacking.

De esta forma, en este trabajo me centro en una parte de la experiencia etnográfica desarrollada en contextos transfeministas de la ciudad de Barcelona entre 2017 y 2018 . Una experiencia que se ha configurado como un encuentro con diferentes miradas críticas, trayectorias, proyectos y prácticas que he interpretado como un proceso político de diálogo y articulación con otros cuerpos, como una experiencia corporal y política etnográfica donde, a través de la participación en diferentes espacios de construcción colectiva y mediante las entrevistas' he vivenciado relaciones intercorporales en torno a las disidencias corporales, sexuales y tecnológicas, así como una articulación e incorporación de las aportaciones y conocimientos como comparto en el apartado final.

Mi trabajo de campo etnográfico forma parte de un proyecto de investigación más amplio, donde indago en las articulaciones entre (trans)feminismos y hacking a partir del hacking corporal, la autonomía tecnológica y la autodefensa digital feminista. En este marco, he identificado que se desarrollan perspectivas, proyectos y prácticas políicas orientadas a reconceptualizar las nociones tradicionales sobre las tecnologías, a la vinculación de las disidencias sexuales, corporales y tecnológicas, así como al desarrollo de posiciones, estrategias y herramientas críticas hacia los ámbitos tecnológicos como sistemas de dominación atravesados por múltiples relaciones de poder, y que apuestan, en consecuencia, por la re-apropiación y autonomía tecnológica.

Desde dichos escenarios, en este apartado profundizo en las prácticas políticas de hacking corporal y retomo la propuesta de Mari Luz Esteban (2004a) acerca de desarrollar enfoques críticos que den cuenta de lecturas, prácticas y resistencias alternativas desde el cuerpo. En este sentido, desde la etnografía me he acercado a trayectorias y proyectos (trans)feministas que, desde mi lectura, se apropian de la perspectiva hacker para activar otras narrativas y prácticas desde las experimentaciones corporales, para hackear los códigos socioculturales normativos de regulación corporal y subjetiva.

A partir de mi experiencia corporal y política etnográfica y de los diálogos intercorporales y las aportaciones de Ixs (trans)feministas entrevistadxs, considero que la experimentación corporal se torna una práctica política para intervenir y modificar la sexualidad, la corporalidad y la subjetividad. Asimismo, el cuerpo se activa como campo de experimentación para diluir las fronteras entre privado-corporal/público-social, para colectivizar las experiencias y producir otras

\footnotetext{
' Entrevistas realizadas con Lucia Egaña, Klau Kinki, Beka Iglesias, Verónica Lahitte, Anamhoo, Ce, Kina, Alex Hache 'Spider Alex', Elena Urko y Diego Marchante 'Gender Hacker' en 2017.
} 
reprogramaciones del género, de las prácticas sexuales, del deseo y del placer, generando, de este modo, conocimientos de resistencia a los modelos socioculturales normativos.

Las prácticas y proyectos activados por Ixs entrevistadxs que toman el cuerpo como espacio de experimentación y producción de conocimientos son diversos y heterogéneos, por lo que en estas líneas destaco los talleres y la postpornografía como dispositivos políticos de experimentación corporal.

Con relación a la postpornografía como dispositivo político de experimentación corporal, considero que ésta se activa desde diferentes prácticas performativas, así como desde diversos proyectos audiovisuales.

Algunos de los proyectos que incorporan prácticas performativas activados por Ixs entrevistadxs con los que he conectado a lo largo del trabajo de campo son los desarrollados por Quimera Rosa ${ }^{2}$ como Sexus 3 y Akelarre Cyborg (junto a Transnoise) ${ }^{3}$ y por Post-op 4.

En cuanto a las prácticas performativas, algunxs de Ixs entrevistadxs como Ce de Quimera Rosa (entrevista personal, 28 de marzo de 2017) y Elena Urko de Post-op (entrevista personal, 18 de octubre de 2017) destacaban como éstas permiten romper la frontera entre lo privado y lo público, lo corporal y social. Así mismo, Kina identifica la performatividad como una práctica central en el trabajo de Quimera Rosa para trabajar con el propio cuerpo e identidad (entrevista personal, 28 de marzo de 2017). En esta misma línea, Urko me compartía su comprensión de la performance como una práctica que incorpora su propia experiencia corporal y proceso vital, así como su propio cuestionamiento del género, la sexualidad y la identidad. Y que, además, al hacerlo público, permite generar imaginarios y representaciones sexuales y corporales alternativas (entrevista personal, 18 de octubre de 2017).

Por su parte, en relación a los proyectos audiovisuales que incorporan la postpornografía como experimentación sobre el propio cuerpo, en estas líneas me gustaría destacar el trabajo de Post-op 5 con proyectos como Implantes, Fantasía PostNuklear, IntroAkto, Atracciones y Nexos ${ }^{6}$, así como el trabajo de Lucía Egaña con la producción del documental Mi Sexualidad es una creación artística (2011)7 sobre las prácticas postpornográficas desde personas y colectivos de la ciudad de Barcelona, y con su participación como organizadora de la Muestra Marrana, un evento autogestionado con varias ediciones dirigido a mostrar producciones audiovisuales relacionadas con prácticas sexuales y cuerpos no normativos y transgresores.

Desde las aportaciones de Elena Urko (entrevista personal, 18 de octubre de 2017) y Lucía Egaña (entrevista personal, 16 de febrero de 2017), estos proyectos posibilitan subvertir imaginarios hegemónicos en torno a la sexualidad y las prácticas sexuales normativas desde trayectorias de disidencia sexual y corporal mediante la apropiación de tecnologías y de códigos audiovisuales.

De esta forma, mediante la performance y lo audiovisual, Lucía Egaña me compartía cómo la postpornografía permite ampliar y modificar las propias prácticas sexuales (entrevista personal, 16 de febrero de 2017), Elena Urko destacaba que la postpornografía le posibilita incorporar sus prácticas contra-sexuales (entrevista personal, 18 de octubre de 2017) y Ce ampliaba la postpornografía como una forma de intervenir el cuerpo y la subjetividad al modificar la propia sexualidad (entrevista personal, 28 de marzo de 2017).

Por otro lado, considero que los talleres son otro dispositivo políico que activa el cuerpo como campo de experimentación y producción de conocimiento que, además, cuenta con una implicación colectiva.

Desde la trayectoria y el trabajo compartido por Ixs entrevistadxs en estas páginas incorporo diversos talleres, como los talleres de GenderHacking ${ }^{8}$ desarrollados por Diego Marchante "Genderhacker' dirigidos a intervenir el género y a actuar y subvertir la masculinidad (entrevista personal, 23 de octubre de 2017). Los talleres sobre prácticas postpornográficas que se realizaban desde Post-op ${ }^{9}$, como los talleres de postporno, de postporno enfocados en la diversidad funcional, los talleres de BDSM o de Ecosex y UrbaSex. Los talleres corporales ${ }^{10}$ activados por Elena Urko, como el Laboratorio sensorial para corporalidades disidentes desarrollado con Patricia Carmona, los talleres de Empoderándonos desde nuestra monstruosidad, Si nos organizamos follamos todes, Empoderamiento corporal y de Ecosex y espacio público. Y los talleres de experimentación corporal con dispositivos electrónicos, sonido y performances colectiva de El cuerpo como instrumento sonoro post-género" de Quimera Rosa (Ce, entrevista personal, 28 de marzo de 2017). Así como

\footnotetext{
2 http://quimerarosa.net/

${ }^{3}$ https://akelarrecyborg.tumblr.com/

${ }^{4}$ https://postop-postporno.tumblr.com/

${ }^{5}$ https://postop-postporno.tumblr.com/videos

${ }^{6}$ https://postop-postporno.tumblr.com/nexos

${ }^{7}$ https://www.lucysombra.org/archives/category/pornologia/mi-sexualidad-es-una-creacion-artistica

${ }^{8} \mathrm{http}: / /$ genderhacker.net/?portfolio=genderhacking

${ }^{9}$ https://postop-postporno.tumblr.com/talleres

${ }^{10} \mathrm{https}$ //elenaurkopostop.com/talleres-disidentes-2/

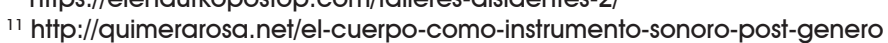


los talleres de biohacking activados por Klau Kinki desde el proyecto de Gynepunk ${ }^{12}$ para la autonomía corporal y la autodefensa ginecológica (entrevista personal, 1 de marzo de 2017) y el workshop Trans ${ }^{*}$ Plant $^{13}$ de Quimera Rosa, desarrollado desde el proyecto Transplant ${ }^{14}$ y dirigido a la modificación de la subjetividad y el cuerpo hacia otras formas no humanas mediante la investigación y experimentación con diversas tecnologías (Kina, entrevista personal, 28 de marzo de 2017).

A partir de las experiencias en los talleres compartidas por Ixs entrevistadxs, considero que son prácticas políticas que operan mediante la experimentación colectiva y posibilitan el intercambio y la construcción colectiva de conocimientos desde las diversas experiencias corporales.

Desde mi punto de vista, tanto la postpornografía como los talleres son prácticas de hacking corporal que materializan la propuesta política desarrollada por Haraway (1995 [1991]), recogida en líneas anteriores, en referencia a las políticas cyborg ubicadas en la parcialidad y el conocimiento situado. Asimismo, en acuerdo con Preciado (2008), dichas políticas de experimentación corporal, que el autor denomina principio de autocobayo, son una forma implícita, situada y corporal de hacer política y producir conocimientos.

\section{Hackear los cuerpos, las tecnologías y los conocimientos como programaciones estandarizadas de código cerrado}

La experiencia política y etnográfica con proyectos transfeministas de hacking corporal me ha orientado a una lectura de los feminismos como prácticas hacker, en la medida en que interpreto que las anteriores prácticas políticas abren, intervienen y subvierten las tecnologías de producción subjetiva y corporal. Asimismo, dicha experiencia me ha llevado a conocer y compartir otras articulaciones entre (trans)feminismos y hacking mediante proyectos y prácticas de autonomía tecnológica y autodefensa digital feminista, como explicitaba al inicio de este apartado.

No obstante, como compartía anteriormente las prácticas que vinculan hacking y (trans)feminismos no se dirigen exclusivamente a decodificar e intervenir el cuerpo y la tecnología, sino que también hackean los códigos y mecanismos hegemónicos de construcción de conocimiento. En este sentido, la experiencia etnográfica me ha potenciado a incorporar perspectivas hacker transfeministas y establecer un paralelismo entre cuerpos, tecnologías y conocimientos como programaciones estandarizadas de código cerrado.

Es decir, la producción y normalización corporal basada en códigos socioculturales puede interpretarse de forma paralela a la producción y control tecnológico regida por el código cerrado y a la producción de conocimiento hegemónica construida desde códigos científicos y académicos.

En acuerdo con Daniela de Araujo (2018), la noción de hack puede ser reapropiada con objeto de activar otras críticas y prácticas para subvertir cualquier sistema. De esta forma, la cultura hacker, Open Source y la filosofía del software y hardware libre, como herramientas para abrir los códigos tecnológicos, las cajas negras de nuestras máquinas y para la emancipación tecnológica, también apuestan por la cultura libre, la apertura de los conocimientos y la construcción de espacios colaborativos, colectivos y horizontales para su producción (Mckenzie WARK, 2005; Eurídice, CABAÑES, 2016).

Por ello, desde mi experiencia política e investigadora interpreto las anteriores prácticas políticas transfeministas de hacking corporal como prácticas críticas a los modelos hegemónicos de construcción del conocimiento mediante la articulación de narrativas, trayectorias, proyectos y redes que enfatizan el carácter político (trans)feminista, las experiencias y experimentación corporal, el intercambio y la construcción colectiva, la horizontalidad y los procesos.

En este sentido, la articulación entre las genealogías teóricas críticas del cuerpo junto con la experiencia etnográfica y los aportes de Ixs entrevistadxs me ha permitido desarrollar una relectura del cuerpo y la producción de conocimiento científico como programación estandarizada de código cerrado, donde vinculo los feminismos, la investigación feminista, la etnografía feminista y los proyectos y prácticas compartidos por Ixs entrevistadxs como genealogías diferenciadas, pero dirigidas a cuestionar y hackear los códigos normativos de producción de conocimiento y a desarrollar herramientas de conocimiento colectivas y transformadoras desde las experiencias corporales.

\section{Reflexiones finales}

Desarrollar una antropología feminista desde los cuerpos, que ponga de relieve las experiencias corporales en los procesos de construcción de conocimientos requiere replantear cuestiones epistemológicas, metodológicas y políticas en el marco de la disciplina.

\footnotetext{
12 https://gynepunk.tumblr.com

${ }^{13} \mathrm{http}: / / q u i m e r a r o s a . n e t /$ transplant/index.php/2018/08/05/transplant-workshopen/

14 http://quimerarosa.net/transplant/
} 
Con el objetivo de plantear propuestas dirigidas a corporeizar las etnografías y a desarrollar una antropología feminista encarnada, en este artículo he destacado los diversos enfoques críticos en los que se inserta mi proceso de investigación, y que me han permitido vivenciar la práctica etnográfica como una experiencia corporal, como un encuentro y proceso de aprendizaje y diálogo con otros cuerpos, a partir del cual se han generado relaciones intercorporales y la incorporación de conocimientos y experiencias.

Considero que reflexionar sobre cómo nos inciden los procesos de investigación que habitamos son cuestiones habitualmente obviadas en la producción científica y académica, incluso desde trabajos orientados por epistemologías y metodologías feministas. Frecuentemente, integramos la subjetividad, la posición situada y la reflexividad, nos interrogamos sobre cómo afectamos a la investigación, pero escasamente nos preguntamos sobre las transformaciones que dichos procesos operan en nosotrxs, sobre lo experimentado e incorporado, aquello que Marisa Ruíz Trejo y Dau García Dauder (2018) denominan epistemología del fuera de campo. Por ello, en este apartado final, me gustaría compartir una reflexión acerca de cómo me ha mutado la investigación etnográfica, constituyéndose en una experiencia de incorporación de conocimientos desde prácticas políticas (trans)feministas de hacking corporal, autonomía tecnológica y autodefensa digital.

En este sentido, la experiencia corporal etnográfica que me ha permitido desarrollar una relectura del cuerpo, la subjetividad, la tecnología y el conocimiento como códigos normativos, e interpretar los (trans)feminismos como perspectivas críticas y prácticas hacking dirigidas a abrir y desvelar dichos códigos para generar formas alternativas de habitar los cuerpos, las subjetividades, las tecnologías y los conocimientos. De este modo, me ha potenciado a revalorizar la relevancia de las prácticas políticas y las experiencias corporales en la producción de conocimientos críticos y de transformación social.

Asimismo, el proceso etnográfico me ha proporcionado una perspectiva feminista sobre las tecnologías y agencia crítica para intervenir mis herramientas y prácticas regidas por corporaciones tecnológicas y modelos socioculturales dominantes. Igualmente, me ha permitido transformar mi relación con las tecnologías mediante la incorporación de conocimientos feministas activistas en mis experiencias cotidianas y prácticas políticas relativos a la autonomía tecnológica y la autodefensa digital.

En este último sentido, la experiencia etnográfica desde las articulaciones entre (trans)feminismos y hacking me ha llevado a compartir desafíos tecnolopolíticos en relación a ampliar las luchas por la autonomía de nuestros cuerpos a nuestras tecnologías, incorporando dichas prácticas en mis experiencias políticas y docentes mediante la participación en proyectos ciberactivistas y ciberfeministas para promover la incidencia política digital de colectivos sociales, la autodefensa digital frente a las ciberviolencias, la autonomía tecnológica y la privacidad y seguridad digital.

\section{Referencias}

ASCHIERI, Patricia; PUGLISI, Rodolfo. "Cuerpo y producción de conocimiento en el trabajo antropológico. Una aproximación desde la fenomenología, las ciencias cognitivas y las prácticas corporales orientales". In: CITRO, Silvia (Coord.). Cuerpos plurales: antropología de y desde los cuerpos. Buenos Aires: Editorial Biblos, 2010. p. 127-148.

AZPEITIA, Marta. "Viejas y nuevas metáforas. Feminismo y filosofía a vueltas con el cuerpo". In: AZPEITIA, Marta; BARRAL, María José; DÍAZ, Lidia Esther; GONZÁLEZ CORTÉS, Teresa; MORENO, Esther; YAGO, Teresa (Eds.). Piel que habla. Viaje a través de los cuerpos femeninos. Barcelona: Icaria, 2001. p. 245-291.

ADÁN, Carme. Feminismo y Conocimiento. De la experiencia de las mujeres al ciborg. Coruña: Espiral Maior Edicions, 2006.

BENTHALL, Jonathan; POLHEMUS, Ted (Eds.). The body as a medium of expression. Nueva York: E.P Dutton, 1975.

BLANCO, Mercedes. "Autoetnografía. Una forma narrativa de generación de conocimientos". Andamios, Ciudad de México, 2012, v. 9, n. 19, p. 49-74. Disponible en https://doi.org/10.29092/ uacm.v9i19.390. Acceso en 18/4/20.

BLACKING, John. The Anthropology of the Body. Londres: Academic Press, 1997.

BLÁZQUEZ, Norma. "Epistemología feminista: temas centrales". In: DA ROSA, Katemari Diogo; CAETANO, Caetano; ALMEIDA (Eds.). Gênero e sexualidade: interseccções necesárias à produção de conhecimentos. Campiña Grande: Realice, 2017. p. 12-31.

BOURDIEU, Pierre. "Notas provisionales para la percepción social del cuerpo". In: VALERA, Julia (Comp.). Materiales de sociología crítica. Madrid: La Piqueta, 1986. p. 183-194. 
BUTLER, Judith [1993]. Cuerpos que importan. Sobre los límites materiales y discursivos del "sexo". Buenos Aires: Paidós, 2002.

BUTLER, Judith [1990]. El género en disputa. Feminismo y la subversión de la identidad. Barcelona: Paidós, 2007.

CABAÑES, Eurídice. "Hackers, software, hardware libre y trabajo colaborativo: la resistencia política del procomún frente al discurso capitalista". In: SORIA, Irene (Ed.). Ética Hacker. Seguridad y Vigilancia. México D.F: Universidad del Claustro de Sor Juana, 2016, p. 67-110.

CITRO, Silvia. "La antropología del cuerpo y los cuerpos en el mundo. Indicios para una genealogía (in)disciplinar". In: CITRO, Silvia (Coord.). Cuerpos plurales: antropología de y desde los cuerpos. Buenos Aires: Editorial Biblos, 2010. p. 17-58

CSORDAS, Thomas J. "Somatic Modes of Attention". Cultural Anthropology, Arlington, 1993, v.8, n.2, p. 135-156. Disponible en https://doi.org/10.1525/can.1993.8.2.02a00010. Acceso en 18/4/20.

CURIEL, Ochy. "Crítica poscolonial desde las prácticas políticas del feminismo antirracista". Nómadas, Bogotá, 2007 n. 26, p. 92-101. Disponible en http://www.redalyc.org/pdf/1051/1051 15241010.pdf. Acceso en 18/4/20.

CURIEL, Ochy [2014]. "Construindo Metodologias Feministas desde o Feminismo Decolonial". In: BALDUINO DE MELO et al. (Orgs.). IV Semana de Reflexões sobre Negritude, Gênero e Raça. Descolonizar o Feminismo. Brasília: Instituto Federal de Educação, Ciência e Tecnologia de Brasília, 2018, p. 32-51.

DE ARAUJO, Daniela. Feminismo e Cultura Hacker: Intersec «Ões entre Política, Gênero e Tecnologia, 2018. Doutorado (Programa de Pós-Graduação em Política Científica e Tecnológica) —Instituto de Geociências de la Universidade Estadual de Campinas, Campinas, Brasil.

DE LAURETIS, Teresa. "Feminist Studies/Critical Studies: Issues, Terms, and Contexts". In: DE LAURETIS, Teresa (Ed.). Feminist Studies/Critical Studies. Bloomington: Indiana University Press, 1986. p. 1-19.

DE LAURETIS, Teresa. Technologies of Gender. Essays on Theory, Film and Fiction. London: Macmillan Press, 1989.

DEL MÁRMOL, Mariana et al. "Entramados convergentes: cuerpo, experiencia, reflexividad e investigación". In: JORNADAS DE SOCIOLOGÍA, V, 2008, La Plata, Universidad de la Plata, 2008. Disponible en http://sedici.unlp.edu.ar/bitstream/handle/10915/59266/Documento_completo_.pdfdes.pdf-PDFA.pdf?sequence=3. Acceso en 18/4/20.

DEL MÁRMOL, Mariana; SÁEZ, Mariana L. "¿De qué hablamos cuando hablamos de cuerpo desde las ciencias sociales?". Question, La Plata, 2011, v. 1, n. 30, p. 1-9. Disponible en http:// perio.unlp.edu.ar/ojs/index.php/question/article/view/1058. Acceso en 20/4/20.

DOUGLAS, Mary [1970]. Símbolos naturales. Exploraciones en cosmología. Madrid: Akal, 1988.

ESPINOSA, Yuderkys. "Una crítica descolonial a la epistemología feminista crítica". El Cotidiano, México D.F, 2014, n. 184, p. 7-12. Disponible en https://www.redalyc.org/pdf/325/32530724004.pdf. Acceso en 20/4/20.

ESPINOSA, Yuderkys; GÓMEZ, Diana; OCHOA, Karina (Eds.). Tejiendo de otro modo: Feminismo, Epistemología y Apuestas descoloniales en Abya Yala. Popayán (Colombia): Universidad del Cauca, 2014.

ESTEBAN, Mari Luz. Antropología del cuerpo. Género, itinerarios corporales, identidad y cambio. Madrid: Anthropos, 2004a.

ESTEBAN, Mari Luz. "Antropología encarnada. Antropología desde una misma". Papeles del CEIC, Bizkaia, v. 12, p. 1-21. Junio, 2004b. Disponible en http://www.ehu.eus/ojs/index.php/papelesCEIC/ article/view/12093. Acceso EN 22/4/2020.

ESTEBAN, Mari Luz. "Cuerpos y políticas feministas: el feminismo como cuerpo". In: VILALBA, Cristina; ÁLVAREZ LUCENA, Nacho (coords.). Cuerpos políticos y agencia. Reflexiones Feministas sobre Cuerpo, Trabajo y Colonialidad. Granada: Universidad de Granada, 2011 . p. 45-84. 
ESTEBAN, Mari Luz; FLORES, Juan Antonio; LÓPEZ, Julián (Coords.). "Incorporaciones Antropológicas. Análisis desde el cuerpo y las emociones". In: CONGRESO DE ANTROPOLOGÍA: LUGARES, TIEMPOS Y MEMORIAS, XII, 2011 , León, FAAEE.

FELIU, Joel. "Nuevas formas literarias para las ciencias sociales: el caso de la autoetnografía". Athenea Digital, Barcelona, 2007, n. 12, p. 162-271. Disponible en https://doi.org/10.5565/rev/ athenead/vOn 12.446. Acceso en 22/4/2020.

FERNÁNDEZ GUERRERO, Olaya. "Pensar con el cuerpo, pensar desde el cuerpo". Thémata. Revista de Filosofía, Logroño, 2012, n. 46, p. 361-368. Disponible en http://www.unirioja.es/genero/archivos/ pdf/comunicacion_pensar_cuerpo.pdf. Acceso en 20/4/2020.

GARCÍA, Noelia. "La autoetnografía. Una experiencia de corporalidad en la investigación sociológica". In: JORNADAS DE JÓVENES INVESTIGADORES, VII, 2013, Buenos Aires, Instituto de Investigaciones Gino Germani, Facultad de Ciencias Sociales, Universidad de Buenos Aires. Disponible en https:// www.aacademica.org/000-076/233.pdf. Acceso en 18/4/2020.

GREGORIO GIL, Carmen. "Contribuciones feministas a problemas epistemológicos de la disciplina antropológica: Representación y relaciones de poder". AlBR. Revista de Antropología Iberoamericana, Madrid, 2006, v. 1, n. 1, p. 22-39. Disponible en https://doi.org/10.11156/ aibr.010104. Acceso en 22/4/2020.

GROSZ, Elizabeth. Volatile Bodies. Toward a Corporeal Feminism. Bloomington: Indiana University Press, 1994.

HARAWAY, Donna. "Situated knowledges: The science question in feminism and the privilege of partial perspective". Feminist studies, Baltimore, v. 14, n. 3, p. 575-599, 1988. Disponible en https:/ /doi.org/10.2307/3178066. Acceso en 20/4/2020.

HARAWAY, Donna [1991]. Ciencia, cyborgs y mujeres. La reinvención de la naturaleza, Madrid: Cátedra, 1995.

HARAWAY, Donna. Modest_Witness@Second_Millenium.FemaleMan@Meets_OncoMouseTm. Feminism and Technoscience. Nueva York: Roülledge, 1997.

HARDING, Sandra [1986]. Ciencia y feminismo. Madrid: Morata, 1996.

HARDING, Sandra (Ed.). Feminism and methodology: Social science issues. Bloomington: Indiana University Press, 1987.

HARDING, Sandra. "¿Existe un método feminista?”. In: BARTRA, Eli (Ed.). Debates en torno a una metodología feminista. México, DF: Universidad Autónoma Metropolitana, 1988. p. 9-34.

HOOKS, bell et al. Otras inapropiables. Feminismos desde las fronteras. Madrid: Traficantes de sueños, 2004.

JACKSON, Michael [1988]. "Conocimiento del cuerpo". In: CITRO, Silvia (Coord.). Cuerpos plurales: antropología de y desde los cuerpos. Buenos Aires: Editorial Biblos, 2010. p. 59-82.

KOSOFSKY SEDGWICK, Eve [1990]. Epistemología del armario. Barcelona: Ediciones La Tempestad, 1998.

KOSOFSKY SEDGWICK, Eve. Tendencies. London: Routledge, 1994.

LAMAS, Marta. Cuerpo: diferencia sexual y género. México: Taurus, 2002.

LUGONES, María. "Towards a decolonial feminist”. Hypatia, v. 25, n. 4, p. 742-759, 2010. doi: https:/ /doi.org/10.1111/j.1527-2001.2010.01137.x.

MARTíN, María Teresa; MUÑOZ, José María. "Epistemología, metodología y métodos. ¿Qué herramientas para qué feminismo? Reflexiones a partir del estudio del cuidado". Quaderns de Psicologia, Barcelona, 2014, v. 16, n. 1, p. 35-44. Disponible en https://doi.org/10.5565/rev/qpsicologia. 1213. Acceso en 18/4/2020.

MAUSS, Marcel [1971]. Sociología y Antropología. Madrid: Editorial Tecnos, 1979.

OKELY, Judith. "Anthropology and autobiography: participatory experience and embodied knowledge". In: OKLEY, Judith; CALLAWAY, Helen (Eds.). Anthropology y Autobiography. Nueva York: Routledge, 1992. p. 1-28. 
PERDOMO REYES, Inmaculada. "Género y tecnologías. Ciberfeminismo y construcción de la tecnocultura actual". Revista Iberoamericana de Ciencia, Tecnología y Sociedad, Buenos Aires/Salamanca, 2016, v. 11, n. 31, p. 171-193. Disponible en http://www.scielo.org.ar/scielo.php?script=sci_abstract\&pid= S1850-00132016000100009. Acceso en 25/4/2020.

PRECIADO, Paul. Testo Yonqui. Madrid: Espasa, 2008.

RUÍZ TREJO, Marisa; GARCÍA DAUDER, Dau. "Los talleres "epistémicos-corporales” como herramientas reflexivas sobre la práctica etnográfica". Universas Humanística, Bogotá, 2018, n. 86, p. 52-88. Disponible en https://doi.org/10.11144/Javeriana.uh86.tech. Acceso en 26/4/2020.

STACEY, Judith [1988]. “Can There Be a Feminist Ethnography?”. In: GLUCK, Sherna; DAPHNE, Patai (Eds.). Women's Words. The Feminist Practices of Oral History. Nueva York: Routledge, 1991 . p. 111-119.

SPIVAK, Gayatri [1985]. “¿Puede hablar el subalterno?”. Revista Colombiana de Antropología, Bogotá, 2003, n. 39, p. 297-374. Disponible en http://www.redalyc.org/pdf/1050/105018181010.pdf. Acceso en 18/4/2020.

SUÁREZ NAVAZ, Liliana; HERNÁNDEZ CASTILLO, Rosalía (Eds.). Descolonizando el feminismo: Teorías y prácticas desde los márgenes. Madrid: Cátedra, 2008.

SCHEPER-HUGHES, Nancy; LOCK, Margaret. "The Mindfull Body: A Prolegomenon to Future Work in Medical Anthropology". Medical Anthropology Quarterly, 1987, v. 1, n. 1, p. 6-41. Disponible en https://doi.org/10.1525/maq.1987.1.1.02a00020. Acceso en 25/7/2020.

TAPALDE MOHANTY, Chandra [1984]. "Bajo los ojos de Occidente. Academia feminista y discurso colonial”. In: SUÁREZ, Liliana; HERNÁNDEZ, Aída (Eds.). Descolonizando el Feminismo: Teorías y Prácticas desde los Márgenes. Madrid: Cátedra, 2008. p. 112-161.

VERGÉS BOSCH, Nuria. Teorías principales de las tecnologías: Evolución y principales debates. Barcelona: Colecciones OMADO, Universidad de Barcelona, 2013. Disponible en: http:// diposit.ub.edu/dspace/handle/2445/45624. Acceso en 26/4/2020.

WARK, McKenzie. Un manifiesto hacker. Barcelona: Alpha Decay, 2005.

WATTS, Jacqueline. "The outsider within': dilemmas of qualitative feminist research within a culture of resistance”. Qualitative Research, 2006, v. 6, n. 3, p. 385-402. Disponible en https://doi.org/10.1177/ 1468794106065009. Acceso en 18/4/2020.

Lola Martínez Pozo (lolamartinezpozo@ugr.es) es activista feminista. Antropóloga y Doctora en Estudios de las Mujeres, Discursos y Prácticas de Género. Investigadora especializada en estudios. de diversidad sexual, corporal y de género; cibercultura, tecnología y feminismos. Personal Docente e Investigador. Grupo de Investigación OTRAS: Perspectivas Feministas en Investigación Social. Instituto Universitario de Estudios de las Mujeres y de Género.

\section{COMO CITAR ESTE ARTíCULO, DE ACUERDO CON LAS NORMAS DE LA REVISTA:}

MARTÍNEZ POZO, Lola. "Corporeizar las etnografías desde perspectivas feministas". Revista Estudios Feministas, Florianópolis, v. 28, n. 3, e58097, 2020.

CONTRIBUCIÓN DE AUTORÍA

No aplicable.

FINANCIACIÓN

Investigación doctoral financiada por el Programa de Contratos Pre-doctorales. Plan Propio de Investigación. Vicerrectorado de Investigación y Transferencia. Universidad de Granada. Desarrollado en el Departamento de Antropología Social y Cultural de la Universidad de Granada, 2015-2019.

CONSENTIMIENTO DE USO DE IMAGEN

No aplicable. 
APROBACIÓN DE COMITÉ DE ÉTICA EN INVESTIGACIÓN

No aplicable.

\section{CONFLICTO DE INTERESES}

No aplicable.

\section{LICENCIA DE USO}

Este artículo está licenciado bajo la Licencia Creative Commons CC-BY Internacional. Con esta licencia se puede compartir, adaptar, crear material para cualquier objetivo, siempre que se le atribuya la autoría.

\section{HISTORIAL}

Recibido el 31/08/2018

Presentado nuevamente el $17 / 04 / 2020$

Aprobado el 15/06/2020 